(९ С.Е. МАМЧУР, И.Н. СИЗОВА, С.А. ШМУЛЕВИЧ, 2014

( ) АННАЛЫ АРИТМОЛОГИИ, 2014

удк 616.124-008.316-073.432.19

DOI: 10.15275/annaritmol.2014.1.4

ОЦЕНКА ВНУТРИЖЕЛУДОЧКОВОЙ ДИССИНХРОНИИ У ПАЦИЕНТОВ С МАНИФЕСТИРУЮЩЕЙ ЖЕЛУДОЧКОВОЙ ПРЕЭКЗИТАЦИЕЙ ПРИ ПОМОЩИ ТРЕХМЕРНОЙ ЭХОКАРДИОГРАФИИ В РЕЖИМЕ РЕАЛЬНОГО ВРЕМЕНИ

Тип статьи: оригинальная статья

С.Е. Мамчур, И.Н. Сизова, С.А. Шмулевич

ФГБУ «Научно-исследовательский институт комплексных проблем сердечно-сосудистых заболеваний» СО РАН; Сосновый б-р, 6, г. Кемерово, 650002, Российская Федерация 
Мамчур Сергей Евгеньевич, доктор мед. наук, заведующий лабораторией, e-mail: mamchse@cardio.kem.ru;

Сизова Ирина Николаевна, канд. мед. наук, ст. научн. сотр.;

Шмулевич Светлана Александровна, заведующий отделением

\begin{abstract}
Цель: оценить внутрижелудочковую диссинхронию у пациентов с манифестирующей желудочковой преэкзитацией при помощи трехмерной эхокардиографии в режиме реального времени (RT3DE).

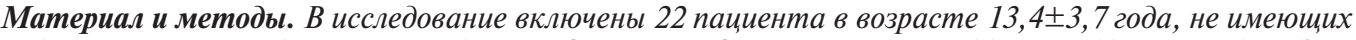
суббективных жалоб. Пациенты были представлены двумя группами: у 11 из них (1-я группа) на ЭКI в покое определялись признаки манифестной преэкзитации, у 11 других (2-я группа) $\Delta$-волна отсутствовала. Определялись стандартное отклонение и максимальная разница интервалов между началом комплекса $Q R S$ и достижением минимального регионарного систолического объема для 16-, 12- и 6-сегментной моделей левого желудочка (ЛЖ).

Результаты. У пациентов с преэкзитацией все изученные показатели были статистически значимо больше, чем у пациентов контрольной группы (без преэкзитации).

Вывод. Манифестирующая желудочковая преэкзитация у бессимптомных пациентов (феномен Вольфа-Паркинсона-Уайта (ВПУ)) приводит к развитию систолической внутрижелудочковой диссинхронии.

Ключевые слова: феномен Вольфа-Паркинсона-Уайта; эхокардиография; диссинхрония.
\end{abstract}

\title{
EVALUATION OF THE INTRAVENTRICULAR DYSSYNCHRONY IN PATIENTS WITH MANIFESTING VENTRICULAR PREEXITATION USING THREE-DIMENSIONAL ECHOCARDIOGRAPHY
}

\section{S.E. Mamchur, I.N. Sizova, S.A. Shmulevich}

Research Institute for Complex Issues of Cardiovascular Diseases, Siberian Branch of the Russian Academy of Sciences; Sosnovyy bul'var, 6, Kemerovo, 650002, Russian Federation

Mamchur Sergey Evgen'evich, MD, DM, Chief of Laboratory, e-mail: mamchse@cardio.kem.ru; Sizova Irina Nikolaevna, MD, PhD, Senior Research Associate; Shmulevich Svetlana Aleksandrovna, Chief of Department

\begin{abstract}
Objective: to evaluate the intraventricular dyssynchrony in patients with manifesting ventricular preexcitation using real-time three-dimensional echocardiography (RT3DE).

Material and methods. 22 patients 13.4 \pm 3.7 years old without subjective complaints were included in the study. 11 of them (group 1) had ECG signs of manifesting preexcitation, other 11 ones (group 2) had no $\Delta$-wave on $E C G$. To assess systolic dyssynchrony the standard deviation and the maximal difference in time from the QRS onset to the minimal regional systolic volume were obtained for 16-, 12-, and the 6-segmental 3D models of left ventricle in each patient.

Results. In patients with ventricular preexcitation all studied parameters had a statistically significant difference with the same ones in control group (without preexcitation).

Conclusion. Manifesting ventricular preexcitation in asymptomatic patients leads to development of the systolic intraventricular dyssynchrony.

Key words: Wolff-Parkinson-White ECG pattern; echocardiography; dyssynchrony.
\end{abstract}

\section{Введение}

$\mathrm{M}$ орфологическим субстратом синдрома Вольфа-Паркинсона-Уайта (ВПУ) является наличие дополнительных путей проведения (ДПП), которые в большинстве случаев электрически шунтируют атриовентрикулярное (AB) соединение [1]. При манифестном синдроме или феномене ВПУ имеется антероградное проведение по ДПП, которое регистрируется на ЭКГ в виде $\Delta$-волны. Механизм ее возникновения - электрическое предвозбуждение части желудочкового миокарда, предшествующее по времени активации желудочков по нормальной проводящей системе [2].
Методом выбора при лечении синдрома ВПУ является радиочастотная аблация (РЧА). Действующие в настоящее время руководства ACC/AHA и ESC [3] относят катетерную РЧА у бессимптомных пациентов с феноменом ВПУ ко ІІа классу показаний, исключение составляют некоторые категории работников (военнослужащие, пилоты, полицейские, спортсмены и т. п.) [4]. Такая консервативная позиция объясняется тем, что традиционно само по себе наличие преэкзитации не рассматривалось как нарушение, которое может приводить к нарушениям систолической и/или диастолической функции сердца. Однако за последние годы в связи с широким внедрением в клиническую 
практику радионуклидных и эхокардиографических методов диагностики стали появляться данные о повышении вероятности развития левожелудочковой дисфункции при манифестировании ДПП [5-8]. Так, продемонстрировано, что наличие манифестных правых септальных и задних парасептальных ДПП может привести к диссинхронии, которая становится причиной ухудшения глобальной систолической функции левого желудочка (ЛЖ) [9, 10].

При эхокардиографической диагностике у пациентов с преэкзитацией во всех вышеперечисленных исследованиях использовались лишь визуальные методы оценки нарушений локальной сократимости ЛЖ, за исключением одного, где был применен метод оценки растяжимости миокарда [8]. Однако в этом исследовании данный метод рассматривался лишь как вариант топической диагностики синдрома ВПУ в сравнении с внутрисердечным картированием. До настоящего времени не предпринимались исследования, в которых бы использовались прямые методы оценки внутрижелудочковой диссинхронии. Факт подтверждения наличия диссинхронии у пациентов с манифестированием антероградного проведения по ДПП может повлиять на пересмотр клинических рекомендаций в пользу более агрессивного подхода у бессимптомных пациентов.

Цель исследования - оценка внутрижелудочковой диссинхронии у пациентов с манифестирующей желудочковой преэкзитацией при помощи трехмерной эхокардиографии в режиме реального времени (RT3DE).

\section{Материал и методы}

В исследование включены 22 пациента в возрасте $13,4 \pm 3,7$ года, не имеющих субъективных жалоб. Пациенты были представлены двумя группами: у 11 из них (1-я группа) на ЭКГ в покое определялись признаки манифестной преэкзитации, у 11 других (2-я группа) $\Delta$-волна отсутствовала. У всех обследованных отсутствовали клинические проявления какого-либо заболевания сердечно-сосудистой системы, и эхокардиография проводилась им в рамках профосмотра. Клиническая характеристика групп пациентов представлена в таблице 1.

ЭКГ-синхронизированная RT3DE выполнялась во время задержки дыхания на ультразвуковом сканере iE33 («Philips Medical Systems», Нидерланды) с использованием трехмерного секторного датчика с фазированной решеткой X3.
Трехмерные изображения сохраняли на жестком диске рабочей станции и в дальнейшем обрабатывали с помощью программного комплекса QLab 8.1 («Philips Medical Systems», Нидерланды). Сегментарную сократимость изучали путем анализа кривых «время-объем» и расчета показателей разброса экскурсии и хронометража для каждого из 16 сегментов ЛЖ по классификации Американской ассоциации эхокардиографии [11].

Для оценки систолической диссинхронии определяли стандартное отклонение интервалов между началом комплекса QRS и моментом достижения минимального регионарного систолического объема для 16-сегментной (Tmsv16-SD), 12-сегментной (Tmsv12-SD) и 6-сегментной (Tmsv6-SD) моделей ЛЖ. Дополнительно определялась максимальная разница между началом QRS и моментом достижения минимального регионарного систолического объема (Tmsv16-Dif, Tmsv12-Dif и Tmsv6-Dif соответственно). Bce вышеназванные индексы диссинхронии нормализовались в виде процентного отношения к длительности интервала R-R (Tmsv16-SD\%, Tmsv12-SD\%, Tmsv6-SD\%, Tmsv16-Dif\%, Tmsv12-Dif\% и Tmsv6-Dif\%). В качестве порогового значения Tmsv16-SD\% был принят уровень $8,3 \%[12,13]$.

Таблица 1

Клиническая характеристика групп пациентов *

\begin{tabular}{lcc|c}
\hline \multicolumn{1}{c|}{ Показатель } & 1 -я группа & 2-я группа & $p$ \\
\hline Возраст, лет & $13(9 ; 17)$ & $12(9 ; 17)$ & 0,921 \\
Пол, м/ж & $7 / 4$ & $6 / 5$ & 0,665 \\
КдР ЛЖ**, мм & $44(40 ; 47)$ & $43(39 ; 46)$ & 0,724 \\
КСР ЛЖ**, мм & $25(22 ; 28)$ & $24(23 ; 27)$ & 0,816 \\
ФВ ЛЖ**, \% & $65(60 ; 68)$ & $66(62 ; 69)$ & 0,307 \\
Переднезадний & $29(25 ; 31)$ & $27(26 ; 30)$ & 0,931 \\
размер ЛП, мм & & & \\
Переднезадний & $18(16 ; 20)$ & $17(16 ; 19)$ & 0,883 \\
размер ПЖ, мм & $7,4(5,9 ; 7,8)$ & $7,3(6,0 ; 7,7)$ & 0,414 \\
Толщина МЖП, мм & $6,8(5,6 ; 7,3)$ & $6,8(5,8 ; 7,2)$ & 0,570 \\
Толщина ЗСЛЖ, мм & $67(66 ; 100)$ & $90(69 ; 104)$ & 0,137 \\
Пик Е, см/с & $80(31 ; 48)$ & $38(32 ; 46)$ & 0,648 \\
Пик А, см/с & $78(62 ; 81)$ & $72(61 ; 79)$ & 0,196 \\
ВИР, мс &
\end{tabular}

* Данные представлены в виде медианы и квартильного размаха.

** Определяли согласно формуле Simpson biplane.

Примечание. КДР - конечный диастолический размер; КСР - конечный систолический размер; ФВ - фракция выброса; МЖП - межжелудочковая перегородка; ЗСЛЖ - задняя стенка левого желудочка; ВИР - время изоволюмического расслабления. 
Статистический анализ проводили в программе Statistica 10 («Statsoft», США), он включал в себя вычисление медиан и квартильных размахов исследуемых величин, а также определение различий по критериям Манна-Уитни и $\chi^{2}$.

\section{Результаты}

Данные ЭКГ-синхронизированной RT3DE в исследованных группах представлены в таблице 2.

На рисунках 1, 2 изображены трехмерные эхокардиограммы и их графический анализ у пациентов, имеющих преэкзитацию и без таковой. Все показатели систолической внутрижелудочковой синхронности у пациента Т. (см. рис. $1, a$ ) сушественно превышают таковые у пациента М. (см. рис. 1, б). У пациента Т. имеется выраженная дисперсия моментов достижения минимального регионарного систолического объема, в то время как у пациента М. эти моменты совпадают по времени. При визуальном анализе как трехмерного изображения ЛЖ, так и сегментарных кривых «время-объем», у пациента Т. визуализируется дискинез 10-го (нижнего среднего) и 15-го (нижнего верхушечного) сегментов.

У пациента Т. (см. рис. 2, a) визуализируется выраженная неоднородность как хронометража, так и экскурсии 10-го, 11-го (средних заднего и заднебокового), 15-го и 16-го (верхушечных заднего и заднебокового) сегментов, что иллюстрируется в виде неоднородного окрашивания на обеих полярных картах и свидетельствует о дис-

Та блица 2

Данные ЭКГ-синхронизированной RT3DE в исследованных группах

\begin{tabular}{l|c|c|c}
\hline \multicolumn{1}{c|}{ Показатель } & 1 -я группа & 2 -я группа & $p$ \\
\hline Tmsv16-SD, мс & $76,5(46,1 ; 118,2)$ & $22,0(10,4 ; 38,5)$ & 0,031 \\
Tmsv12-SD, мс & $62,3(39,5 ; 93,5)$ & $20,5(12,6 ; 32,3)$ & 0,048 \\
Tmsv6-SD, мс & $48,0(26,3 ; 78,5)$ & $17,5(10,4 ; 28,7)$ & 0,009 \\
Tmsv16-Dif, мс & $116,3(81,9 ; 174,6)$ & $43,3(17,0 ; 72,6)$ & 0,008 \\
Tmsv12-Dif, мс & $103,5(68,0 ; 143,8)$ & $35,7(19,3 ; 53,8)$ & 0,012 \\
Tmsv6-Dif, мс & $66,8(32,9 ; 109,4)$ & $27,3(14,1 ; 46,6)$ & 0,046 \\
Tmsv16-SD, \% & $10,2(6,3 ; 13,4)$ & $2,9(1,4 ; 4,3)$ & 0,025 \\
Tmsv12-SD, \% & $8,3(5,4 ; 10,6)$ & $2,7(1,7 ; 3,6)$ & 0,033 \\
Tmsv6-SD, \% & $6,4(3,6 ; 8,9)$ & $2,3(1,4 ; 3,2)$ & 0,018 \\
Tmsv16-Dif, \% & $15,5(11,2 ; 19,8)$ & $5,7(2,3 ; 8,1)$ & 0,041 \\
Tmsv12-Dif, \% & $13,8(9,3 ; 16,3)$ & $4,7(2,6 ; 6,0)$ & 0,027 \\
Tmsv6-Dif, \% & $8,9(4,5 ; 12,4)$ & $3,6(1,9 ; 5,2)$ & 0,891 \\
R-R, мс & $750(731 ; 882)$ & $759(741 ; 896)$ & \\
\hline
\end{tabular}

* Данные представлены в виде медианы и квартильного размаха.
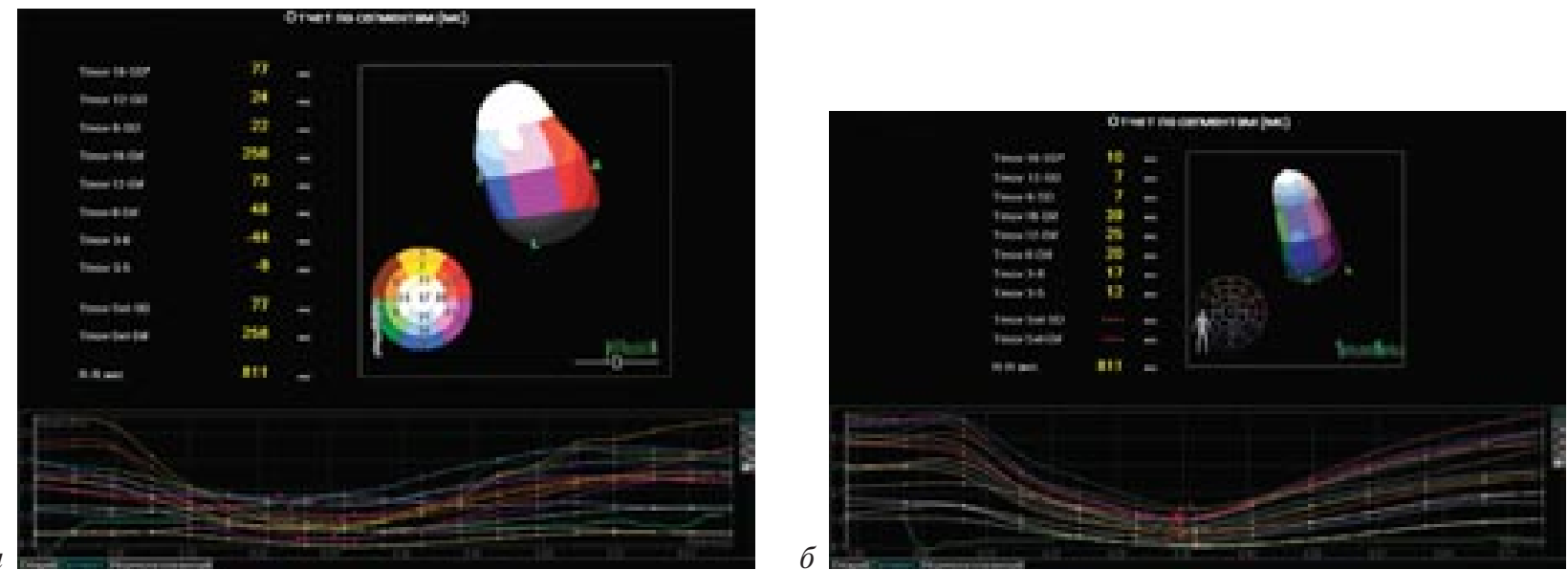

Рис. 1. Трехмерная эхокардиография пациента Т. с манифестной преэкзитацией (a) и пациента М., не имеющего преэкзитации (б), в виде 16-сегментной модели 

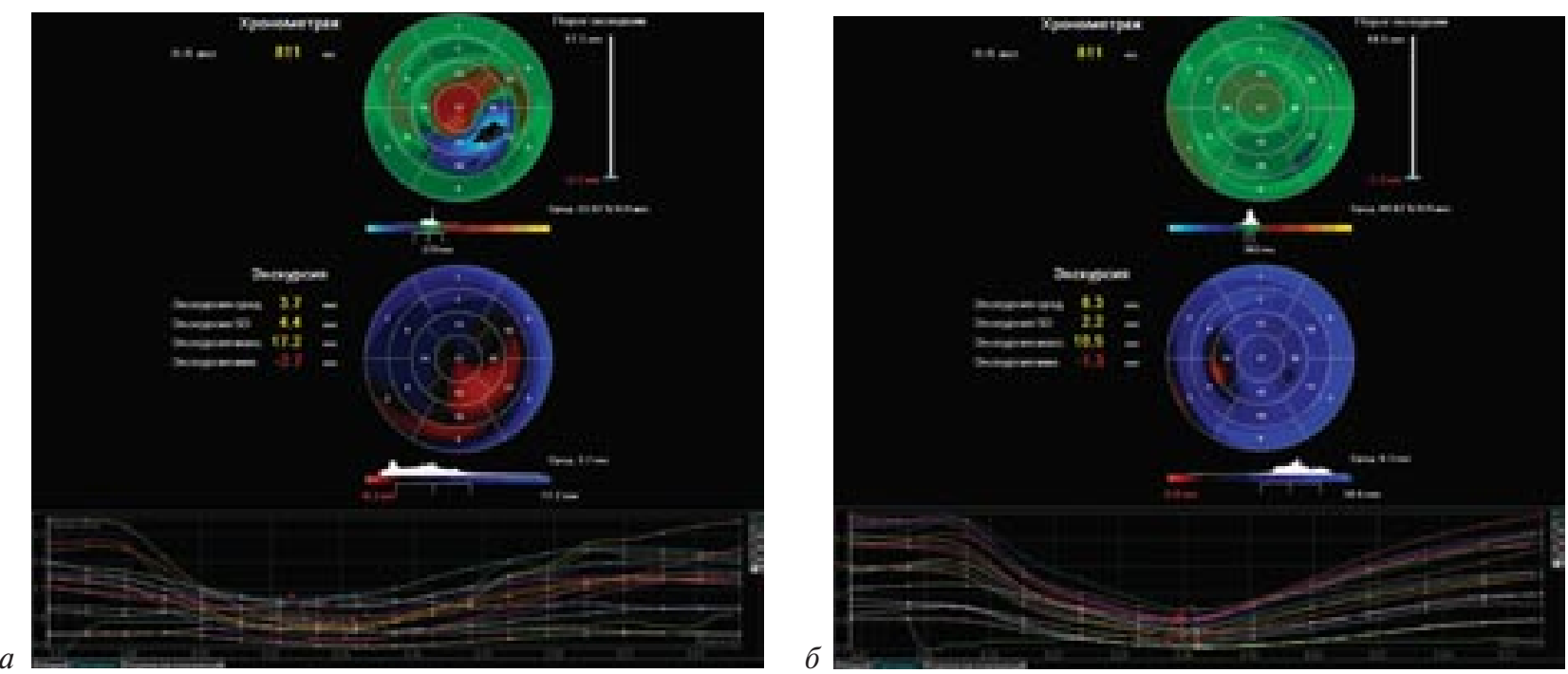

Рис. 2. Анализ хронометража и амплитуды экскурсии сегментов у тех же больных - пациента Т. $(a)$ и пациента М. (б) на модели полярного картирования срезов по короткой оси, расположенных концентрически от верхушки к основанию сердца (также известного как «бычий глаз»). На картах хронометража синие оттенки соответствуют более раннему сокращению, красные - более позднему. На картах экскурсии синие оттенки соответствуют нормокинезу, красные - дискинезу

синхронии. У пациента М. (см. рис. 2, б) имеется гораздо меньшая дисперсия экскурсии за счет верхушечного перегородочного сегмента (вариант нормы) и почти отсутствует дисперсия хронометража, что иллюстрируется в виде однородного окрашивания полярной карты хронометража и свидетельствует об отсутствии диссинхронии.

В результате анализа полученных данных выявлено, что у пациентов с преэкзитацией все изученные показатели оказались статистически значимо больше, чем у пациентов контрольной группы (без преэкзитации). Эти результаты свидетельствуют о том, что у пациентов с феноменом ВПУ имеются инструментально подтвержденные данные о наличии систолической внутрижелудочковой диссинхронии, в отличие от пациентов, не имеющих преэкзитации, у которых признаки диссинхронии отсутствуют.

\section{Обсуждение}

В настоящем исследовании на основании данных трехмерной эхокардиографии в режиме реального времени продемонстрировано наличие систолической внутрижелудочковой диссинхронии у пациентов при бессимптомном наличии манифестной желудочковой преэкзитации (феномена ВПУ). При этом у обследованных значение стандартного отклонения интервалов между началом комплекса QRS и моментом достижения минимального регионарного систолического объема для 16-сегментной модели ЛЖ превышало общепринятый диагностический порог диссинхронии.

K настоящему времени накоплено небольшое количество знаний о том, что при этом «доброкачественно» протекающем состоянии, во многих случаях не требующем лечения, может наблюдаться различного рода механическая дисфункция миокарда как предсердий, так и желудочков, в том числе межжелудочковая диссинхрония [9].

Последняя, по данным ряда исследователей, может послужить единственной причиной ухудшения систолодиастолической функции сердца, вплоть до развития дилатационной кардиомиопатии, в том числе и при феномене ВПУ [14-17]. Сравнительно недавно И.А. Хамнагадаевым и соавт. [6] было показано, что патологическая последовательность возбуждения желудочков при манифестном синдроме и феномене ВПУ может приводить к нарушению диастолической функции, в том числе правого желудочка, задолго до развития систолической дисфункции. Выполнение таким пациентам РЧА ДПП приводит к восстановлению диастолических нарушений. Эти факты, как и результаты нашего исследования, свидетельствуют о необходимости учета эхокардиографических параметров при определении показаний к РЧА, а также необходимости пересмотра действующих в настоящее время рекомендаций в сторону более агрессивного подхода у бессимптомных пациентов. 
Ограничением настоящего исследования является небольшой объем наблюдений, что не позволяет получить статистически значимые данные относительно вклада в диссинхронию ДПП различных локализаций и различной степени предвозбуждения. Также в дальнейшем планируется провести исследование о влиянии РЧА манифестных ДПП на динамику диссинхронии.

\section{Вывод}

Манифестирующая желудочковая преэкзитация у бессимптомных пациентов (феномен ВПУ) приводит к развитию систолической внутрижелудочковой диссинхронии.

\section{Конфликт интересов}

Конфликт интересов не заявляется.

\section{Библиографический список}

1. Klein G.J., Yee R., Sharma A.D. Longitudinal electrophysiologic assessment of asymptomatic patient with the Wolff-Parkinson-White electrocardiographic pattern. N. Engl. J. Med. 1989; 320: 1229-33. DOI: 10.1056/ NEJM198905113201901.

2. Chugh A., Bogun F., Morady F. Catheter ablation of accessory pathways. In: Wilber D.J., Packer D.L., Stevenson W.G. (eds). Catheter ablation of cardiac arrhythmias: basic concepts and clinical applications. 3rd ed. New York-Malden: Blackwell Futura; 2008: 149-72.

3. Blomstrom-Lundqvist C., Scheinman M.M., Aliot E.M. et al. ACC/AHA/ESC guidelines for the management of patients with supraventricular arrhythmias - executive summary: a report of the American College of Cardiology/American Heart Association Task Force on Practice Guidelines and the European Society of Cardiology Committee for Practice Guidelines (Writing Committee to Develop Guidelines for the Management of Patients With Supraventricular Arrhythmias). Circulation. 2003; 108 (15): 1871-909. DOI: 10.1161/ 01.CIR.0000091380.04100.84

4. Steinbeck G. Should radiofrequency current ablation be performed in asymptomatic patients with the Wolff-Parkinson-White syndrome? Pacing Clin. Electrophysiol. 1993; 16 (3, Pt 2): 649-52.

5. Марцинкевич Г.И., Соколов А.А., Ковалев И.А. и др. Электромеханическое сопряжение миокарда в норме и у детей с синдромом ВПУ. Вестник аритмологии. 2004; 35: 38-44.

6. Хамнагадаев И.А., Школьникова М.А., Коков Л.С. и др. Диастолическая функция правого желудочка у больных с манифестным синдромом и электрокардиографическим феноменом Вольфа-Паркинсона-Уайта. Вестник аритмологии. 2012; 68: 21-7.

7. Chen C., Li D., Miao C. et al. LV dyssynchrony as assessed by phase analysis of gated SPECT myocardial perfusion imaging in patients with Wolff-Parkinson-White syndrome. Eur. J. Nucl. Med. Mol. Imaging. 2012; 39 (7): 1191-8. DOI: 10.1007/s00259-012-2101-4.

8. Delelis F., Lacroix D., Richardson M. et al. Two-dimensional speckle-tracking echocardiography for atrioventricular accessory pathways persistent ventricular pre-excitation despite successful radiofrequency ablation. Eur. Heart J. Cardiovasc. Imaging. 2012; 13 (10): 840-8. DOI: 10.1093/ehjci/jes048.

9. Kwon B., Bae E., Kim G. et al. Septal dyskinesia and global left ventricular dysfunction in pediatric Wolff-Parkinson-White syndrome with septal accessory pathway. J. Cardiovasc. Electrophysiol. 2010; 21 (3): 290-5. DOI: 10.1111/ j.1540-8167.2009.01612.x.
10. Tomaske M., Janousek J., Rãzek V. et al. Adverse effects of Wolff-Parkinson-White syndrome with right septal or posteroseptal accessory pathways on cardiac function. Europace. 2008; 10 (2): 181-9. DOI: 10.1093/europace/eun005.

11. Laskey W.K., Pennell D.J., Rumberger J.A. et al. American Heart Association Writing Group on myocardial Segmentation and Registration for Cardiac Imaging: Standardized myocardial segmentation and nomenclature for tomographic imaging of the heart. A statement for healthcare professionals from the Cardiac Imaging Committee of the Council on Clinical Cardiology of the American Heart Association. Circulation. 2002; 105 (4): 539-42. DOI: 10.1161/hc0402.102975.

12. Dai M., Lu J., Qian D.-J. et al. Assessment of left ventricular dyssynchrony and cardiac function in patients with different pacing modes using real-time three-dimensional echocardiography: Comparison with tissue Doppler imaging. Exp. Ther. Med. 2013; 6 (5): 1213-9. DOI: 10.3892/etm.2013.1292.

13. Kapetanakis S., Kearney M.T., Siva A. et al. Real-time threedimensional echocardiography: a novel technique to quantify global left ventricular mechanical dyssynchrony. Circulation. 2005; 112 (7): 992-1000. DOI: 10.1161/ CIRCULATIONAHA.104.474445.

14. Cadrin-Tourigny J., Fournier A., Andelfinger G., Khairy P. Severe left ventricular dysfunction in infants with ventricular preexcitation. Heart Rhythm. 2008; 5 (9): 1320-2. DOI: 10.1016/j.hrthm.2008.05.022.

15. Emmel M., Balaji S., Sreeram N. Ventricular preexcitation associated with dilated cardiomyopathy: a causal relationship? Cardiol. Young. 2004; 14 (6): 594-9. DOI: 10.1017/ S1047951104006031.

16. Fazio G., Mongiovi M., Sutera L. et al. Segmental dyskinesia in Wolff-Parkinson-White syndrome: a possible cause of dilatative cardiomyopathy. Int. J. Cardiol. 2008; 123 (2): 31-4. DOI: 10.1016/j.ijcard.2006.11.109.

17. Iwasaku T., Hirooka K., Taniguchi T. et al. Successful catheter ablation to accessory atrioventricular pathway as cardiac resynchronization therapy in a patient with dilated cardiomyopathy. Europace. 2009; 11 (1): 121-3. DOI: 10.1093/europace/ eun318.

\section{References}

1. Klein G.J., Yee R., Sharma A.D. Longitudinal electrophysiologic assessment of asymptomatic patient with the Wolff-Parkinson-White electrocardiographic pattern. N. Engl. J. Med. 1989; 320: 1229-33. DOI: 10.1056/ NEJM198905113201901.

2. Chugh A., Bogun F., Morady F. Catheter ablation of accessory pathways. In: Wilber D.J., Packer D.L., Stevenson W.G. (eds). Catheter ablation of cardiac arrhythmias: basic concepts and clinical applications. 3rd ed. New York-Malden: Blackwell Futura; 2008: 149-72.

3. Blomstrom-Lundqvist C., Scheinman M.M., Aliot E.M. et al. ACC/AHA/ESC guidelines for the management of patients with supraventricular arrhythmias - executive summary: a report of the American College of Cardiology/American Heart Association Task Force on Practice Guidelines and the European Society of Cardiology Committee for Practice Guidelines (Writing Committee to Develop Guidelines for the Management of Patients With Supraventricular Arrhythmias). Circulation. 2003; 108 (15): 1871-909. DOI: 10.1161/ 01.CIR.0000091380.04100.84.

4. Steinbeck G. Should radiofrequency current ablation be performed in asymptomatic patients with the Wolff-Parkinson-White syndrome? Pacing Clin. Electrophysiol. 1993; 16 (3, Pt 2): 649-52.

5. Martsinkevich G.I., Sokolov A.A., Kovalev I.A. et al. Electromechanical coupling of myocardium in healthy persons and in pediatric patients with WPW-syndrome. Vestnik Aritmologii. 2004; 35: 38-44 (in Russian).

6. Khamnagadaev I.A., Shkol'nikova M.A., Kokov L.S. et al. Diastolic function of the right ventricle in patients with both manifesting syndrome and electrocardiographic phenomen of Wolf-Parkinson-White. Vestnik Aritmologii. 2012; 68: 21-7 (in Russian).

7. Chen C., Li D., Miao C. et al. LV dyssynchrony as assessed by phase analysis of gated SPECT myocardial perfusion imaging in 
patients with Wolff-Parkinson-White syndrome. Eur. J. Nucl. Med. Mol. Imaging. 2012; 39 (7): 1191-8. DOI: 10.1007/s00259-012-2101-4.

8. Delelis F., Lacroix D., Richardson M. et al. Two-dimensional speckle-tracking echocardiography for atrioventricular accessory pathways persistent ventricular pre-excitation despite successful radiofrequency ablation. Eur. Heart J. Cardiovasc. Imaging. 2012; 13 (10): 840-8. DOI: 10.1093/ehjci/jes048.

9. Kwon B., Bae E., Kim G. et al. Septal dyskinesia and global left ventricular dysfunction in pediatric Wolff-Parkinson-White syndrome with septal accessory pathway. J. Cardiovasc. Electrophysiol. 2010; 21 (3): 290-5. DOI: 10.1111/ j.1540-8167.2009.01612.x.

10. Tomaske M., Janousek J., Rãzek V. et al. Adverse effects of Wolff-Parkinson-White syndrome with right septal or posteroseptal accessory pathways on cardiac function. Europace. 2008; 10 (2): 181-9. DOI: 10.1093/europace/eun005.

11. Laskey W.K., Pennell D.J., Rumberger J.A. et al. American Heart Association Writing Group on myocardial Segmentation and Registration for Cardiac Imaging: Standardized myocardial segmentation and nomenclature for tomographic imaging of the heart. A statement for healthcare professionals from the Cardiac Imaging Committee of the Council on Clinical Cardiology of the American Heart Association. Circulation. 2002; 105 (4): 539-42. DOI: 10.1161/hc0402.102975.
12. Dai M., Lu J., Qian D.-J. et al. Assessment of left ventricular dyssynchrony and cardiac function in patients with different pacing modes using real-time three-dimensional echocardiography: Comparison with tissue Doppler imaging. Exp. Ther. Med. 2013; 6 (5): 1213-9. DOI: 10.3892/etm.2013.1292.

13. Kapetanakis S., Kearney M.T., Siva A. et al. Real-time threedimensional echocardiography: a novel technique to quantify global left ventricular mechanical dyssynchrony. Circulation. 2005; 112 (7): 992-1000. DOI: 10.1161/ CIRCULATIONAHA.104.474445.

14. Cadrin-Tourigny J., Fournier A., Andelfinger G., Khairy P. Severe left ventricular dysfunction in infants with ventricular preexcitation. Heart Rhythm. 2008; 5 (9): 1320-2. DOI: 10.1016/j.hrthm.2008.05.022.

15. Emmel M., Balaji S., Sreeram N. Ventricular preexcitation associated with dilated cardiomyopathy: a causal relationship? Cardiol. Young. 2004; 14 (6): 594-9. DOI: 10.1017/S1047951104006031.

16. Fazio G., Mongiovi M., Sutera L. et al. Segmental dyskinesia in Wolff-Parkinson-White syndrome: a possible cause of dilatative cardiomyopathy. Int. J. Cardiol. 2008; 123 (2): 31-4. DOI: 10.1016/j.ijcard.2006.11.109.

17. Iwasaku T., Hirooka K., Taniguchi T. et al. Successful catheter ablation to accessory atrioventricular pathway as cardiac resynchronization therapy in a patient with dilated cardiomyopathy. Europace. 2009; 11 (1): 121-3. DOI: 10.1093/europace/eun318.

Поступила 15.07.2014 г. Подписана в печать 20.07.2014 г. 